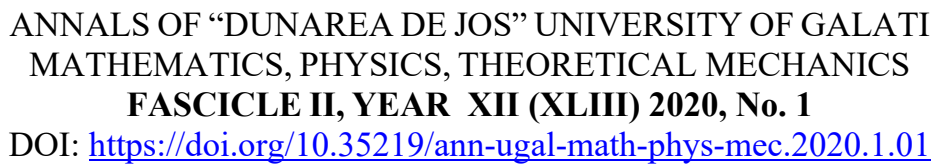

\title{
Multiple closely spaced scatterers location based MUSIC via inverse scattering amplitude estimation
}

\author{
Maria (Stan) Necula ${ }^{1,2,3}$, Dorin Bibicu ${ }^{3,4,5}$, Luminita Moraru ${ }^{1,3^{*}}$, Cristian-Victor- \\ Eugen Rusu ${ }^{6}$ \\ 1 "Dunarea de Jos" University of Galati, Faculty of Sciences and Environment, Department of Chemistry, Physics \\ and Environment, 800008 Galati, Romania \\ 2 Technical College 'Radu Negru' Galati, Romania; 800170 Galati, Romania \\ 3 "Dunarea de Jos" University of Galati, Faculty of Sciences and Environment, Department of Modelling \& \\ Simulation Laboratory, 800008 Galati, Romania \\ 4 "Dunarea de Jos" University of Galati, Faculty of Economics and Business Administration; Department of Business \\ Administration, 800008 Galati, Romania \\ 5 High School Dunărea, Romania; 800402 Galati, Romania \\ 6 "Dunarea de Jos" University of Galati, 111 Domneasca St., 800008 Galati, Romania \\ *Corresponding author: luminita.moraru@ugal.ro
}

\begin{abstract}
This study is an attempt to answer questions related to a new scanning technology applicable in nondivest and noncontact conditions. MUltiple SIgnal Classification (MUSIC) algorithm uses an array of $\mathrm{N}$ transceivers to assess the location of closely spaced scatterers placed in various geometries. MUSIC algorithm works together with the Foldy-Lax (FL) formulation of the full multiple scattering model and the distorted-wave Born approximation (DWBA) model. The experiments were carried out in noisy conditions in order to highlight the noise robustness of the MUSIC algorithm and the capability of its approximations in target location. The average visibility index over the wavelength range was employed to evaluate the accuracy and stability of the FL and DWBA approximations. The FL formulation provides signal reconstruction for target location with fair accuracy and motivates the requirement for a new scanning technology.
\end{abstract}

Keywords: inverse scattering; MUSIC algorithm; Foldy-Lax formulation; distorted-wave Born approximation; average visibility index

\section{INTRODUCTION}

In this study, the MUltiple SIgnal Classification (MUSIC) method is used for locating a finite number of small scatterers embedded in an arbitrary background medium or in a medium with known properties [1-4]. An inverse scattering problem establishes the nature of an inhomogeneity such as the location, geometry, and/or material property, viewed as a scatterer, by using the knowledge of the scattered field. A large amount of data related to inverse scattering and MUSIC has been widely used in ultrasound and microwave applications.

According to the findings reported in [5], this problem can be seen as a scattering problem in which incident plane waves scatter off one or more impenetrable objects. MUSIC uses the so-called 'singular value decomposition of the multistatic scattering matrix' technique (SVD). SVD is independent of 'the composition of the scatterers to be analysed' and is dependent only on the background Green function $\mathrm{G}_{0}\left(\mathrm{r}, \mathrm{r}^{\prime}\right)$ and on the source distribution $[1,6]$. SVD makes it possible to 
decompose a matrix A (size $\mathrm{n} \times \mathrm{d}$ ) into a sum of rank-one matrices by a factorisation in the left and right singular vectors, respectively, and a diagonal matrix whose entries are the singular values of A. The MUSIC algorithm uses two approximations: the Foldy-Lax (FL) formulation of the multiple scattering model [7-9] and the distorted-wave Born approximation model (DWBA) [9-15]. DWBA is only relevant at small incident angles and at small exit/reflection angles. The Foldy-Lax approximation for scattering by a collection of small obstacles provides an approximation of the farfields as an asymptotic expansion which allows us to distinguish between close placed obstacles from those which are placed away from each other. Here, the self-interactions at the target locations are excluded. Nevertheless, in recent decades, only simple geometries were analysed, mainly in the frequency range of the electromagnetic field [9-11].

In our case, we are focusing on the data collected at 5,10,50, and $100 \mathrm{~Hz}$ in order to develop a new scanning technology applicable in a nondivest and noncontact condition. To our knowledge, results in these frequency ranges have not been reported in the literature. Normalised wavelengths of $1,0.5,0.1$, and 0.05 are addressed, ensuring the condition in which the scatterers are much smaller than the wavelength is respected. Additive white Gaussian noise (AWGN) having a variation of 0.0009 and 0.09 is used to investigate the noise immunity. Similarly, the noise-free case is analysed for comparison. The simulation results are presented and discussed relative to the four scatterers' spatial distribution geometries. An estimation of the scattering amplitudes provided by the MUSIC pseudospectrum is performed. The average visibility index over the wavelength range is evaluated for the best reconstruction solution.

\subsection{Helmholtz equation}

\section{EXPERIMENTAL}

For a time-harmonic acoustic wave $\mathrm{U}(\boldsymbol{y}, \mathrm{t})=\operatorname{Re}\left\{\mathrm{n}(\mathrm{y}) \mathrm{e}^{-\mathrm{dat}}\right\}$ with frequency $\omega$, the space-dependent part ${ }^{\mathrm{w}(\mathrm{g})}$ satisfies the Helmholtz equation in the space-frequency domain $(\mathrm{g}, \mathrm{w})$

$$
\Delta \mathrm{u}\left(\mathrm{I}_{2} \omega\right)+\mathrm{k}^{2}\left(\mathrm{I}_{2} \omega\right) \mathrm{u}\left(\mathrm{I}_{2} \omega\right)=\mathrm{f}(\mathrm{I}, \omega)
$$

in $\mathbb{R}^{\mathfrak{a}} \mathbf{D}$, where $\mathrm{D}$ denotes an object that is situated in a homogeneous isotropic medium with density $\mathrm{a}$ and speed of sound c. $r$ is the position vector of any point in the propagation space. $a(y, \omega)$ is the total field produced by the distribution of sources $f\left(x_{,} \propto\right)$. The transceivers are placed in $\kappa_{\mathrm{k}, \mathrm{k}} \mathrm{k}-1, \ldots \mathrm{N}$. $v^{2}(x w)-v_{0}^{2}\left(w, w-v(w, w)\right.$, where $k_{0}^{2}$ is the known wavenumber of the background medium, and $\checkmark\left(y_{,} \omega\right)$ is the scattering potential of the targets to be computed, which is defined for $M$ targets as

$$
\mathrm{v}(\mathrm{r}, \omega)=\sum_{m=1}^{M} \tau_{m} \delta\left(\mathrm{r}-\mathrm{x}_{\mathrm{m}}\right)
$$

where $x_{\mathrm{mn}}, \mathrm{m}=1,2, \ldots, \mathrm{M}$ are the target positions (to be reconstructed); $\tau_{\mathrm{m}} \in \mathrm{C}, \mathrm{m}=1,2, \ldots, \mathrm{M}$ are known target scattering strengths; and $\partial(D)$ is Dirac's delta function. The target scattering strengths is the scattering coefficient for the $\mathrm{m}$-th scatterer, which characterizes how strongly the scatterer interacts with incident waves and can be determined from enforcing energy conservation. Starting from this point, the variable $\omega$ is ignored with the meaning that the results hold for a given frequency.

\subsection{Inverse scattering problem formulation 2.2.1 Foldy-Lax formulation}

The FL formulation provides a model for the scattered wave field when a collection of $\mathrm{M}$ point scatterers located at $x_{\mathrm{rn}}, \mathrm{m}=1,2, \ldots, \mathrm{M}$ is considered at a particular frequency $\omega$ that is still suppressed [12-14]. The total field is computed as the sum of the scattered field and the incident field for the kth transceiver as follows: 


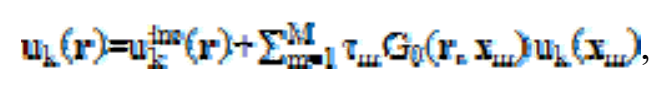

where the target scattering strength $\tau_{m}=0$ for the $\mathrm{m}^{\text {th }}$ point scatterer is known, and $G_{0}$ is the freespace/background Green function. In the scatterer locations, $r=x_{\mathrm{rn}}$ and the background Green function is singular:

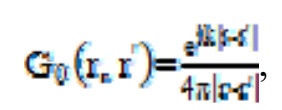

The Green function significance is an outgoing wave observed at $\mathrm{I}$ that is produced by a point source placed at $\mathrm{r}^{\prime}$.

If the self-interactions at the target locations are excluded, then the FL formulation of (3) is

$$
v_{\mathrm{k}}\left(\mathrm{x}_{\mathrm{m}}\right)=\mathrm{u}_{\mathrm{k}}^{\mathrm{m}}\left(\mathrm{x}_{\mathrm{m}}\right)+\sum_{\mathrm{m}=\mathrm{m}^{\prime}} \mathrm{s}_{\mathrm{m}} \mathrm{G}_{\mathrm{g}}\left(\mathrm{x}_{\mathrm{m}}, \mathrm{x}_{\mathrm{m}}\right) \mathrm{u}_{\mathrm{k}}\left(\mathrm{x}_{\mathrm{m}}\right)
$$

The FL framework consists of a set of M coupled linear equations with the known data on the incident field $u_{k}^{i n s}$, scatterer locations $x_{\mathrm{rn}}$, and target scattering strength $\tau_{\mathrm{m} \text {. }}$. In matrix form, (3) becomes

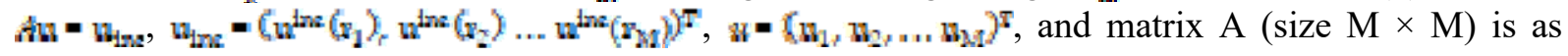
follows:

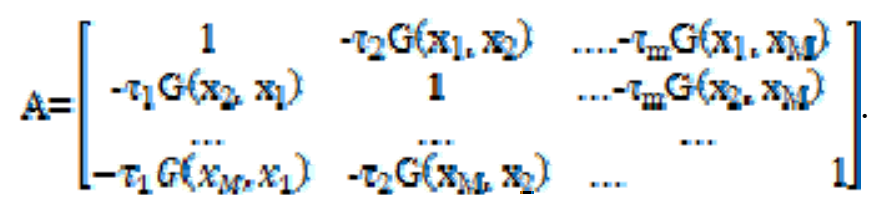

Based on Green's representation theorem and assuming a coincident array in the low-frequency acoustic field, we can consider that the incident waves $w_{k}^{\text {tme }}, \mathbb{k}-1, \ldots N_{r}$, are equal to the background Green function $G_{q}\left(y_{2}, \sigma_{\mathrm{k}}\right)$ evaluated for a source point $\mathrm{r}^{\prime}=\alpha_{\mathrm{z}}$ at a transmitter location. Furthermore, the target outputs are equal to a complete Green function $\left.C_{(2,}, \sigma_{\mathrm{k}}\right)$ of the combined background with the addition of the target medium for a source point $r^{\prime}=\alpha_{h}$ at the kth emitter and, similarly, $G\left(\alpha_{h}, r\right)$ for the jth receiver location. A coincident array meets the condition $J-k-\mathbb{N}$, and (3) and (5) can be rewritten as

$$
\begin{aligned}
& G\left(1, \alpha_{k}\right)-G_{0}\left(r_{i} \alpha_{l}\right)+\sum_{m=1}^{M} \tau_{m} G_{0}\left(r_{i} x_{m}\right) G\left(x_{m}, \alpha_{k}\right) \\
& G\left(x_{m}, \alpha_{1}\right)=G_{0}\left(x_{m}, \alpha_{2}\right)+\sum_{m=m} \tau_{m} G\left(x_{m}, x_{m}\right) G\left(x_{m}, \alpha_{2}\right)
\end{aligned}
$$

When different transceiver elements are considered in (7), the matrix formulation (6) allows for building the so-called multistatic scattering matrix in the FL approximation, which is shown to be symmetric due to the reciprocity of the complete Green functions:

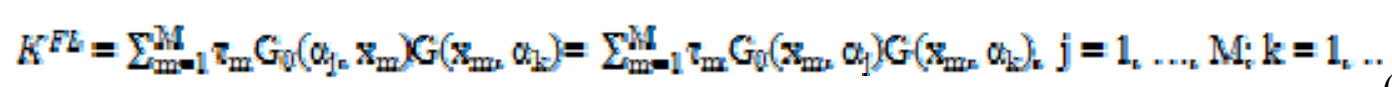


The matrix formulation (9) is a correction of Green's function accounting for the scattering of considered targets.

\subsubsection{Distorted-wave Born approximation (DWBA) model}

When the scattering targets are characterised as weak scatterers, the DWBA model can be used to analyse the distorted wave $[9,15]$. DWBA does not consider multiple scattering effects between the targets of interest and has been successfully applied to a given region where homogeneous scatterers exist. This model is quite limited for inhomogeneous scatterer distributions. If the scattering targets show acoustic properties different from the background medium, then the DWBA approximation can be used:

$$
G\left(x_{m,} \alpha_{1}\right)=G_{0}\left(x_{m k} \alpha_{1}\right)
$$

The multistatic scattering matrix within the DWBA becomes

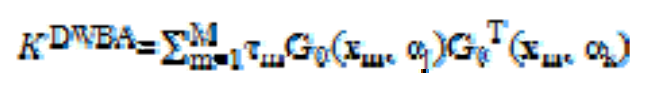

The complete Green function does not appear in DWBA, and the only unknowns are the target locations $\mathbf{x}_{\mathrm{m}}$. DWBA estimates the target locations from the multistatic scattering matrix when the condition $\mathrm{M} \& \mathrm{~N}$ is met.

\subsection{MUSIC algorithm}

The MUSIC algorithm decomposes an observed signal into signal and noise components, and uses the properties of the signal vectors to be orthogonal to the noise vectors. MUSIC operates in the noise subspace based on the Green function with a high spatial resolution of the reconstructed objects; the phase patterns provided by the Green function are the mean of the identifying positions.

Let there be $M$ point scatterers located at $x_{m a}=\left\{x_{1} \ldots x_{M}\right\} \in \mathbb{R}^{5}$. According to the results reported by Kirsch [16], the MUSIC algorithm is based on the SVD of K matrix and uses the discrete version of the multistatic scattering matrix (9) and (11) to define the wave scattering amplitude. When $\mathrm{N} \geq \mathrm{M}$, based on the $\mathrm{K}$ matrix, two matrices $\mathrm{s}_{\mathrm{jm}}, \mathrm{j}=1, \ldots, \mathrm{N}$ and $\mathrm{m}=1, \ldots, \mathrm{M}$ consisting of the background

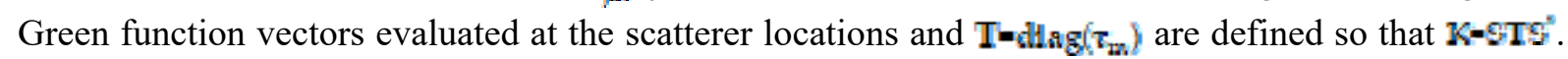
$S^{*}$ is the adjoint of S. MUSIC characterises the range of a self-adjoint operator. As mentioned in [16], when $\mathrm{N} \geq \mathrm{M}$ and if the location of the point scatterers ensures the condition that matrix $\mathrm{S}$ has maximal rank $\mathrm{M}$, then matrices $\mathrm{K}$ and $\mathrm{S}$ coincide.

Further, the problem of estimating the locations of targets follows the signal subspace method proposed by Marengo and Gruber [17]. A signal subspace $\mathrm{S}$ and noise subspace $\mathrm{N}$ are defined. Let us consider the eigenvalues and related eigenvectors of the $\mathrm{K}$ matrix. When the eigenvectors correspond to significant eigenvalues, then the $\mathrm{S}$ signal subspace is spanned. When the corresponding eigenvectors have eigenvalues smaller than the noise level, they span the noise subspace. Let us consider $\wp$ as the projection of matrix K onto the noise subspace. According to [18-20], a simpler and faster mathematical approach is to consider a steering vector $\boldsymbol{f}(\boldsymbol{x})$ with the property $f\left(\boldsymbol{f} f(\boldsymbol{x})=0\right.$. By plotting the pseudospectrum function $\frac{1}{\mid\{\rho f(x) \mid}$, MUSIC is able to determine the locations of the scatterers, as sharp peaks in the MUSIC algorithm do not request data in the shape of the small scatterers. The pseudospectra defined by MUSIC in the DWBA approximation use the background Green function vectors evaluated at the scatterer locations, $x_{1}, \ldots x_{M}-\{\mathrm{f} f(\mathrm{x})$ while the pseudospectra in the FL model are related to the complete Green function.

\subsection{Selection algorithm}


The target spatial distribution is dependent on the experimental design. We studied the target location problem for four spatial distribution geometries. The MUSIC algorithm is implemented for small noise $\mathrm{n}_{1}$ and a large amount of noise $\mathrm{n}_{2}$ that will disturb the detection of the scatterers. The algorithm used to select the higher signal amplitudes $\mathcal{A}$, is as follows:

Step 1: Define the set of parameters as $p\{1, n 1, n 2\}$, where $n_{1}$ and $n_{2}$ denote AWGNs having a variance of 0.0009 and 0.09 , respectively;

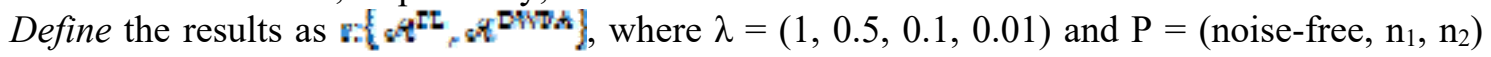
(see Table 1);

Step 2: Generate the possible combinations, the experimental parameters and results are distributed

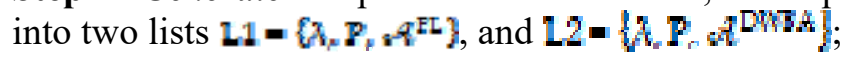

Step 3: Extract the highest amplitude $\mathcal{A}$ values from last two lists L1 and L2 and generate the $\mathcal{A}$ list $\left\{\mathcal{A}^{\mathrm{iL}}, \mathcal{A}^{\mathrm{DWBa}}\right\}$ (see data in Tables 2 and 3 );

Step 4: Run steps 2 and 3 for 16 times for each target distribution; 64 measurements are made.

\subsection{Average visibility index}

The average visibility index $\Upsilon$ is used as a quantitative characteristic of the representation of the MUSIC pseudospectrum. The accuracy of the reconstructed scattering amplitude for the scatterers placed in various spatial distributions with sizes smaller than the wavelength is evaluated based on the so-called average visibility index, as follows [22]:

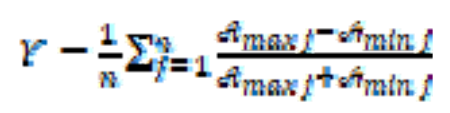

A denotes the amplitude provided by the MUSIC pseudospectrum, indexes 'max' and 'min' correspond to the maximum and minimum amplitudes and $n$ is the number of simulations. $Y$ ranges from 0 to 1 .

\subsection{Simulation framework}

\section{RESULTS AND DISCUSSION}

The simulation approach was implemented in the MATLAB 2017b environment [21]. The simulation results were obtained for a nondispersive background model consisting of a uniform and constant medium velocity. Six scatterers were placed at different locations. The propagation space was considered a rectangular grid $(\mathrm{x}, \mathrm{z})$. The acoustic wave propagation was directed downward toward the negative $\mathrm{z}$. The propagation space grid was dynamically correlated to the wavelength values in order to ensure the condition that the size of the scatterers is much smaller than the wavelength. Both the noise-free and noisy cases were considered.

The following parameters were used:

- $\quad \mathrm{N}=10$ transceivers in a coincident linear array;

- $\quad M=6$ closely spaced scatterers having a spatial distribution into triangle (T), parallelogram $(\mathrm{P})$, diamond (D), and ellipse (E) geometries;

- In order to transform the wavelength values into a range from 0 to 1 , we normalize them by dividing the current value by the maximum value. Normalised wavelengths are $\lambda=1,0.5,0.1$, and 0.05 ;

- AWGN with variances of 0.0009 and 0.09 is added;

\subsection{Results}

Figure 1 displays plots of the chosen discrete locations. The transceiver array is symmetrical and is located at $(-20,0),(-15,0),(-10,0),(-5,0)$, and $(-2,0)$. The targets are located as follows: triangle $(\mathrm{T})$ : $(0,-8),(-1,-9),(1,-9),(-2,-10),(0,-10)$, and $(2,-10)$; parallelogram $(\mathrm{P}):(-2,-8),(0,-8),(2,-8),(0,-9),(2,-$ $9)$, and $(4,-9)$; diamond (D): $(0,-6),(-2,-8),(0,-10),(2,-8),(0,-7)$, and $(0,-9)$; and ellipse (E): $(0,-8),(-$ 
$3,-9),(3,-9),(-3,-10),(3,-10)$, and $(0,-11)$. All positions are relative to wavelength $\lambda$. The target separations are $\lambda, \sqrt{2} \lambda$, and $2 \lambda$. The target scattering strength is $\tau=(1,1.3,1.6,1.6,1.3,1)$.

Some examples of the pseudospectrum and scatterers detection are shown in Figures 2 and 3 for a triangle spatial distribution. Figure 4 plots the results of scatterers detection in noisy case $\mathrm{n}_{2}$ for the diamond and ellipse spatial distributions.

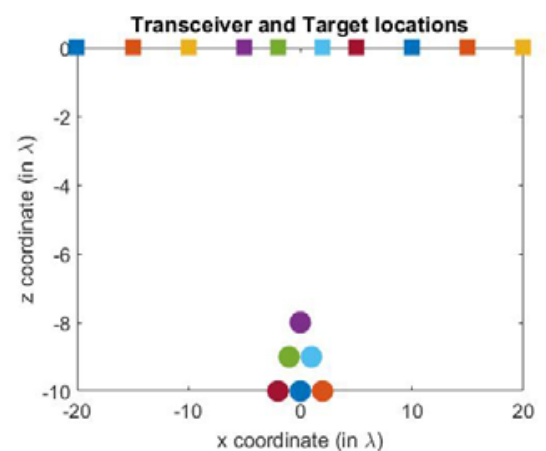

a)

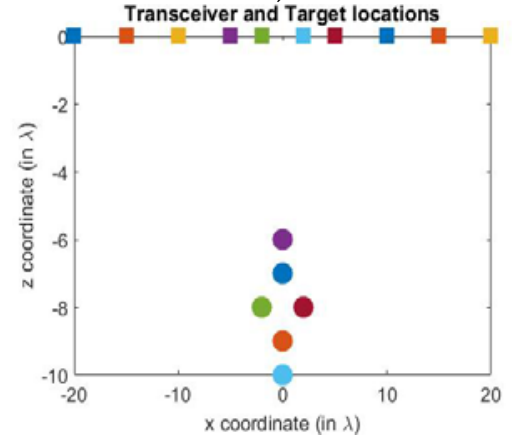

c)

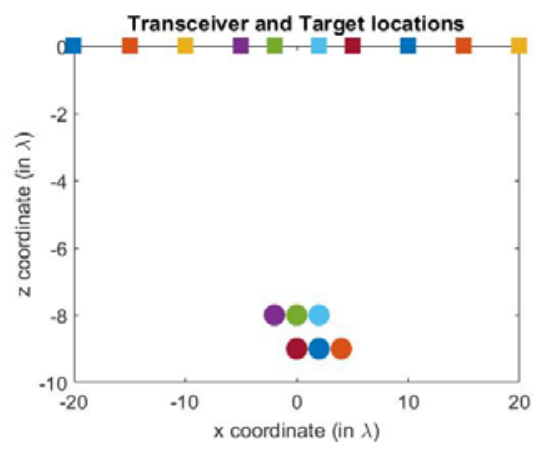

b)

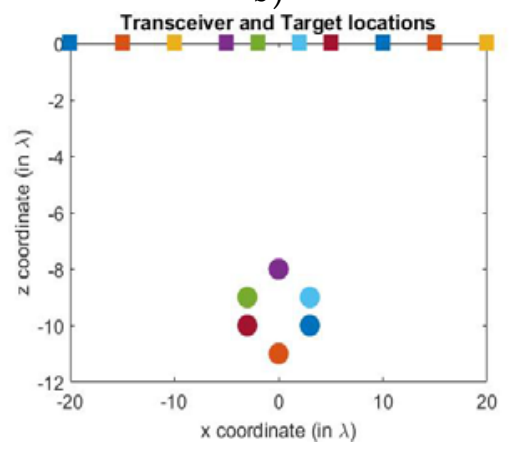

d)

Fig 1. 10 transceivers (depicted as squares) and 6 targets (depicted as circles). The spatial distributions are represented as a) triangle $(T)$, b) parallelogram $(P), c)$ diamond $(D)$, and d) ellipse (E).

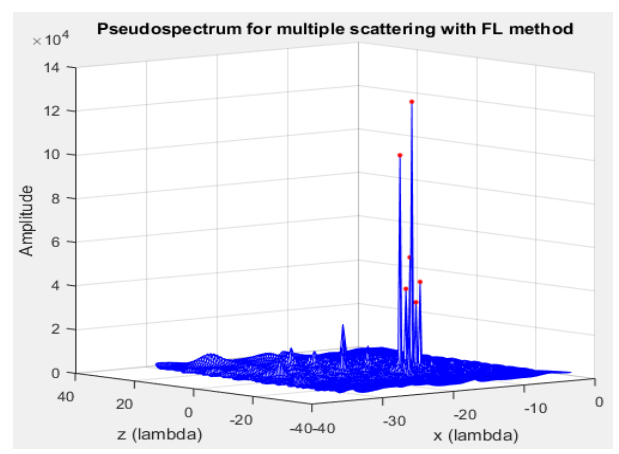

a)

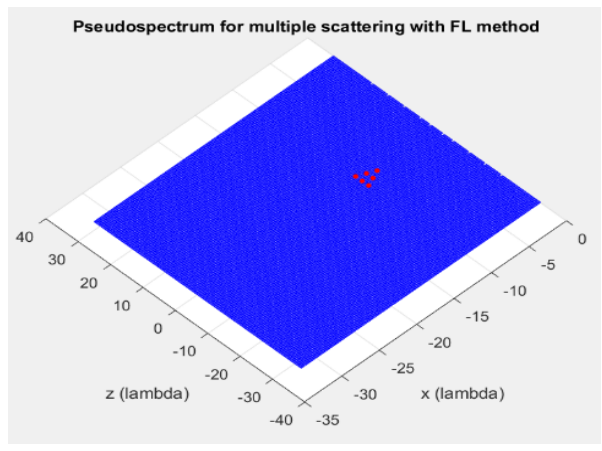

b) 


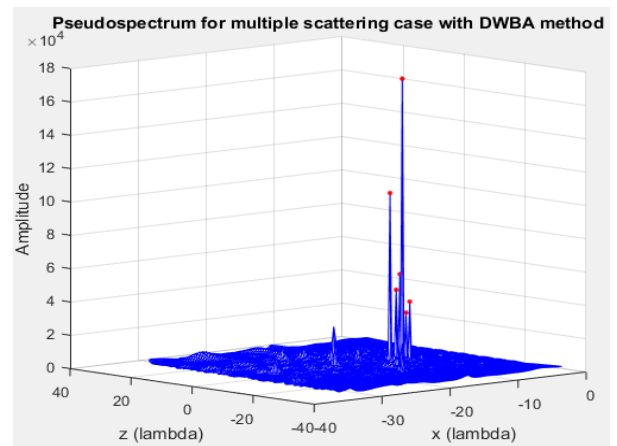

c)

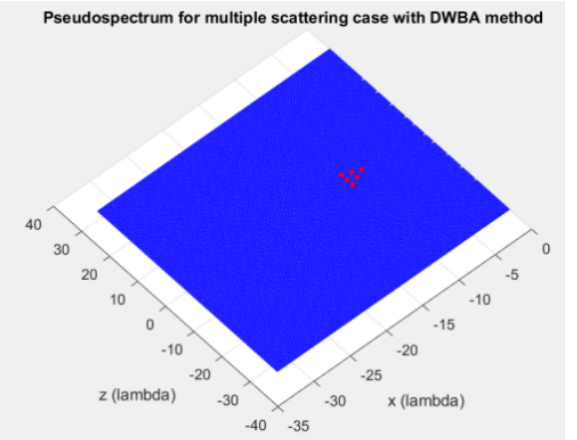

d)

Fig 2. MUSIC pseudospectrum and targets detection for $3 D$ (left column) and $2 D$ (right column) visualisation for $n_{1}, \lambda=1$, and triangle (T). $a-b$ ) FL approximation and $\left.c-d\right) D W B A$ approximation.

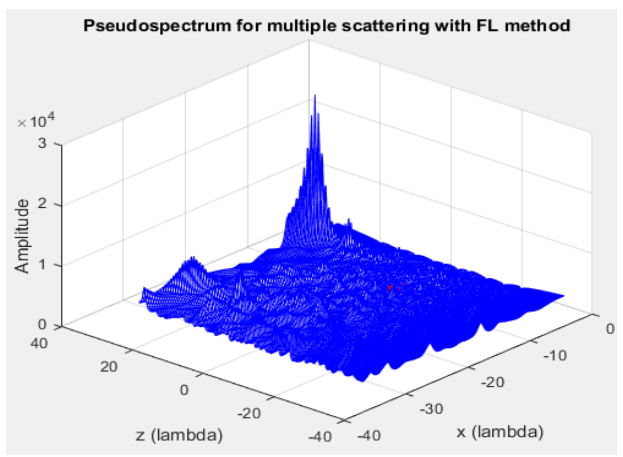

a)

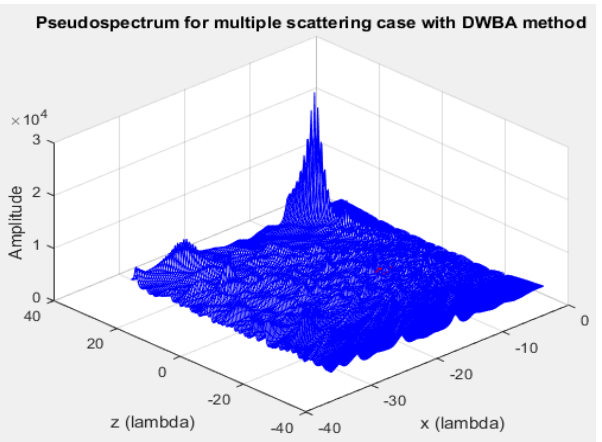

c)

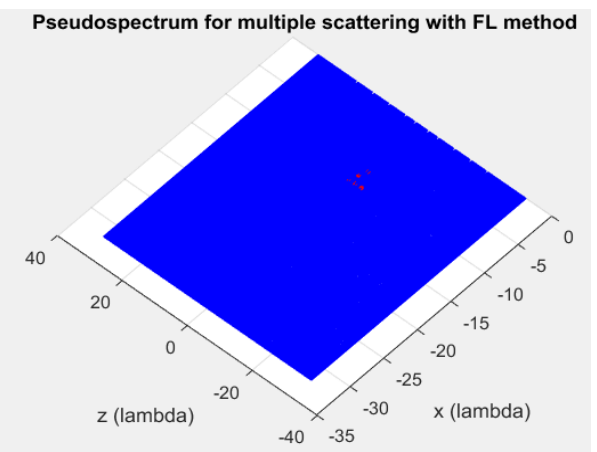

b)

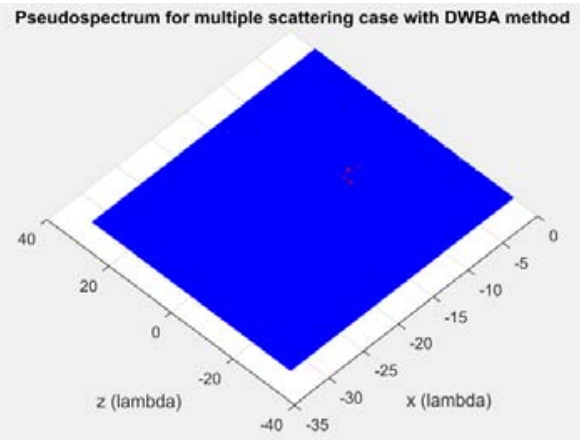

d)

Fig 3. MUSIC pseudospectrum and targets detection for $3 D$ (left column) and $2 D$ (right column) visualisation for $n_{2}, \lambda=1$, and triangle (T). $\left.a-b\right) F L$ approximation and $\left.c-d\right) D W B A$ approximation. 


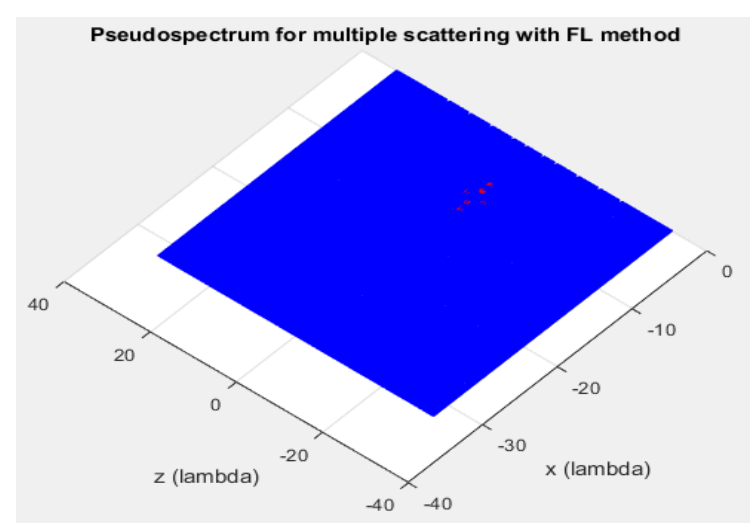

a)

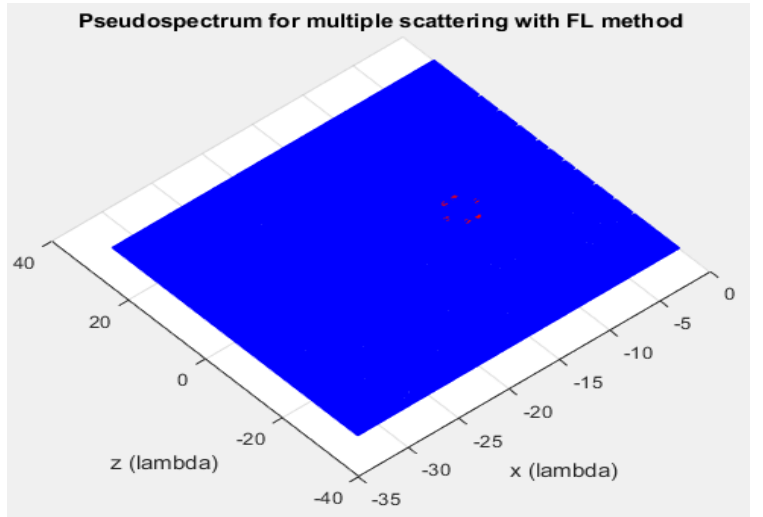

C)

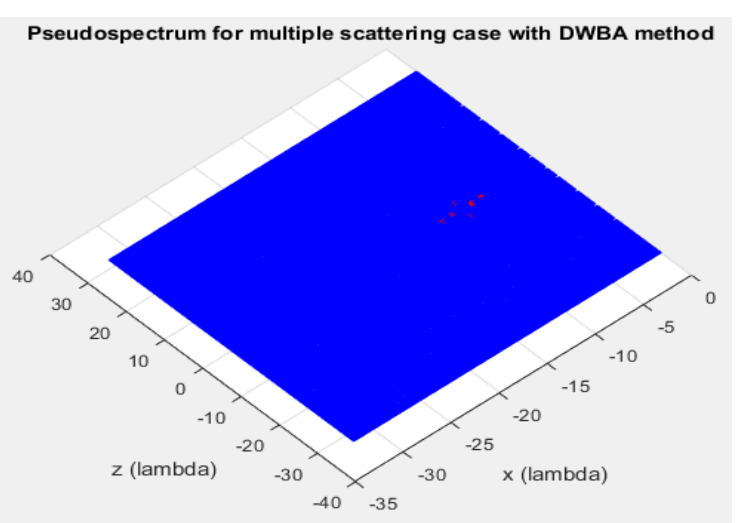

b)

Pseudospectrum for multiple scattering case with DWBA method

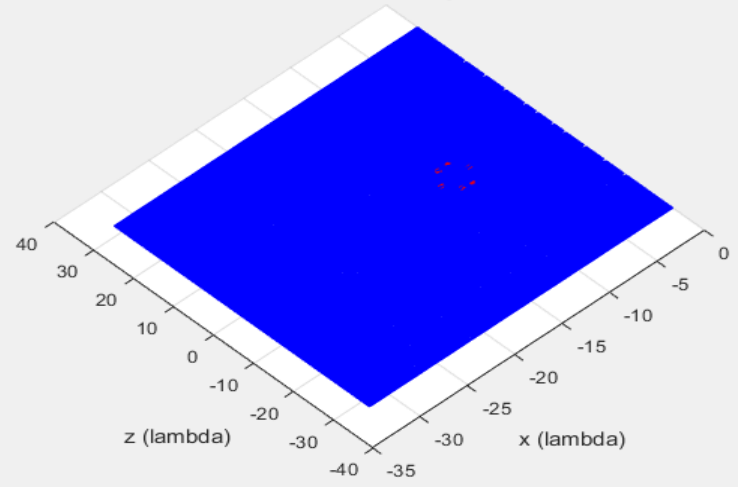

d)

Fig 4. Scatterers detection for diamond (top line) and ellipse (bottom line) in noisy case $n_{2}$ for FL and DWBA approximations.

According to the data presented in Table 1, an improvement of generally $10 \%$ in signal amplitudes $(\mathcal{A})$ of the pseudospectra is achieved. As expected, the case of noise-free scattering returns the same results.

Table 1. Average amplitude ( $A$ ) of scattered signals attained in simulation experiments for $2 D$ obstacles, extracted from list $L 1$ and $L 2$, for FL and DWBA approximations.

\begin{tabular}{|c|c|c|c|c|c|}
\hline Geometry & $\begin{array}{c}\text { AWGN } \\
\text { noise }\end{array}$ & $\begin{array}{l}\text { SNR } \\
\text { (dB) }\end{array}$ & $\lambda$ & $A^{\mathrm{FL}}$ & $\mathcal{A}^{\text {DWBA }}$ \\
\hline \multirow{4}{*}{ (T) } & $\mathrm{n} 1$ & 22.38 & $\begin{array}{c}1 \\
0.5\end{array}$ & 72073 & 63082 \\
\hline & & & $\begin{array}{c}0.1 \\
0.05\end{array}$ & 13173 & 12729 \\
\hline & $\mathrm{n} 2$ & 6.3 & $\begin{array}{c}1 \\
0.5\end{array}$ & 480 & 469 \\
\hline & & & $\begin{array}{c}0.1 \\
0.05\end{array}$ & 187 & 177 \\
\hline \multirow[b]{2}{*}{ (P) } & $\mathrm{n} 1$ & 22.66 & $\begin{array}{c}1 \\
0.5\end{array}$ & 97896 & 91591 \\
\hline & & & $\begin{array}{c}0.1 \\
0.05\end{array}$ & 44955 & 43475 \\
\hline
\end{tabular}




\begin{tabular}{|c|c|c|c|c|c|}
\hline Geometry & $\begin{array}{c}\text { AWGN } \\
\text { noise }\end{array}$ & $\begin{array}{l}\text { SNR } \\
\text { (dB) }\end{array}$ & $\lambda$ & $\mathcal{A}^{\mathrm{FL}}$ & $\mathcal{A}^{\mathrm{DWBA}}$ \\
\hline \multirow{3}{*}{ (D) } & $\mathrm{n} 2$ & 8.9 & $\begin{array}{c}1 \\
0.5 \\
0.1 \\
0.05\end{array}$ & 191 & 187 \\
\hline & $\mathrm{n} 1$ & 22.12 & $\begin{array}{c}1 \\
0.5 \\
0.1 \\
0.05\end{array}$ & $\begin{array}{l}85708 \\
69885\end{array}$ & $\begin{array}{l}77328 \\
61827\end{array}$ \\
\hline & $\mathrm{n} 2$ & 3.3 & $\begin{array}{c}1 \\
0.5 \\
0.1 \\
0.05\end{array}$ & $\begin{array}{l}687 \\
524\end{array}$ & 676 \\
\hline (E) & $\mathrm{n} 2$ & 9.32 & $\begin{array}{c}1 \\
0.5 \\
0.1 \\
0.05 \\
1 \\
0.5 \\
0.1 \\
0.05\end{array}$ & 30912 & 24563 \\
\hline
\end{tabular}

The data presented in Tables 2 and 3 shows examples of the highest selected amplitude of the reconstructed signals $\mathcal{A}$ extracted from L1 and L2. In a noisy environment, FL provides an efficient solution for the inverse scattering problem. In addition, the amplitude is much easier to acquire in the simulation and facilitates target localization. Diamond (D) and ellipse (E) distributions have larger amplitude values in a noisy environment. For all target spatial configurations, better results were obtained at the normalised wavelengths $\lambda=1$ and 0.5 .

Table 2. Highest $\boldsymbol{A}^{F L}$ results across various spatial distributions and wavelengths for AWGN noise-added $\left(n_{1}\right.$ and $\left.n_{2}\right)$ and noise-free values.

\begin{tabular}{|ccccccccc|}
\hline \multicolumn{3}{|c}{ Noise-free } & \multicolumn{4}{c}{$\mathbf{n}_{\mathbf{1}}$} & \multicolumn{3}{c|}{$\mathbf{n}_{\mathbf{2}}$} \\
$\boldsymbol{\lambda}$ & Geometry & ${ }_{\boldsymbol{A}} \boldsymbol{A}^{\mathrm{FL}}$ & $\boldsymbol{\lambda}$ & Geometry & $\boldsymbol{A}^{\mathrm{FL}}$ & $\boldsymbol{\lambda}$ & Geometry & $\boldsymbol{A}^{\mathrm{FL}}$ \\
\hline 0.5 & (D) & $8.76 \cdot 10^{+33}$ & 0.5 & $(\mathrm{P})$ & $6.95 \cdot 10^{+6}$ & 1 & $(\mathrm{D})$ & 932 \\
0.5 & (D) & $8.76 \cdot 10^{+33}$ & 0.5 & (E) & $4.74 \cdot 10^{+6}$ & 1 & $(\mathrm{~T})$ & 1009 \\
\hline
\end{tabular}

Table 3. Highest DWBA results across various spatial distributions and wavelengths for AWGN noise-added (n1 and n2) and noise-free values.

\begin{tabular}{|ccccccccc|}
\hline \multicolumn{3}{c}{ Noise-free } & \multicolumn{4}{c|}{$\mathbf{n}_{1}$} & \multicolumn{3}{c|}{$\mathbf{n}_{2}$} \\
$\lambda$ & Geometry & $\mathscr{A}^{\text {DWBA }}$ & $\lambda$ & Geometry & $\mathcal{A}^{\text {DWBA }}$ & $\lambda$ & Geometry & $\mathcal{A}^{\text {DWBA }}$ \\
1 & (D) & $4.68 \cdot 10^{+33}$ & 0.5 & $(\mathrm{E})$ & $6.01 \cdot 10^{+6}$ & 1 & (D) & 915 \\
0.5 & (D) & $4.68 \cdot 10^{+33}$ & 0.5 & $(\mathrm{E})$ & $4.00 \cdot 10^{+6}$ & 1 & $(\mathrm{~T})$ & 992 \\
\hline
\end{tabular}




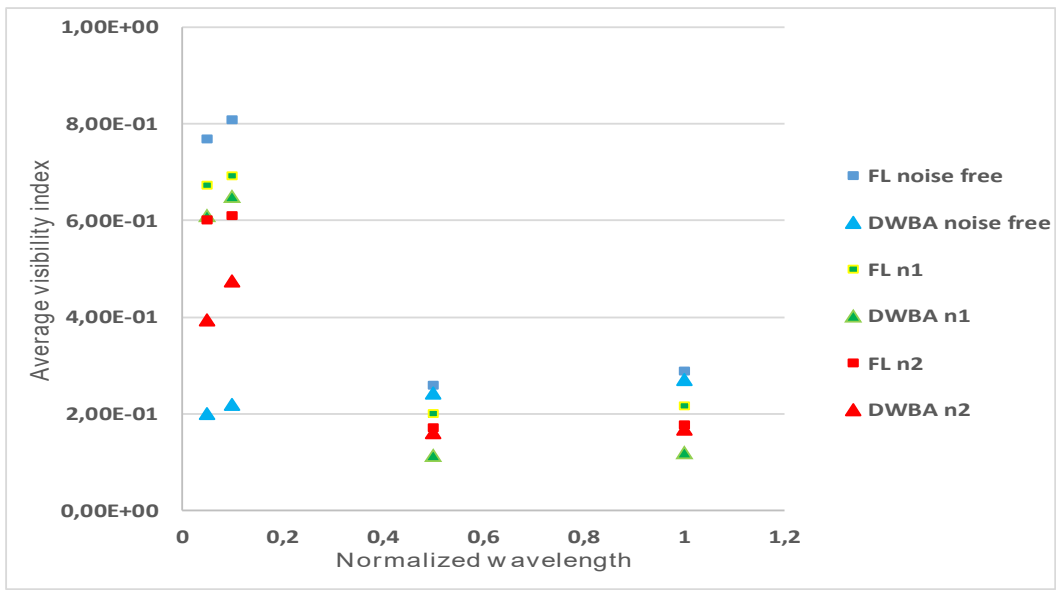

Fig 5. Average visibility index $\mathrm{Y}$ distribution over normalised wavelength.

The average visibility index $\Upsilon$ distribution over the wavelength is presented in Fig. 5. We find significant differences between the backscatter amplitudes with approximations and noise conditions. The reconstruction effect is provided with fair accuracy for pseudospectra provided by the FL approximation with larger $Y$ values.

\subsection{Discussion}

The MUSIC algorithm provides information on the target locations using the poles in the pseudospectrum. Once the target locations are reconstructed from the poles in the MUSIC pseudospectrum, the unknown target scattering amplitudes $A$ are computed by using the values of these locations in equations (9) and (11) and solving the resulting system of equations. The scattering amplitudes $A$ measurements at a fixed frequency were used as performance indicators. The identified poles of the MUSIC pseudospectrum will peak at the same target locations but with different lobe structure and amplitude $A$.

The data displayed in Figures 3 and 4 clearly indicates the influence of noise on the scattered field. For a noisy simulation framework (AWGN noise $\mathrm{n}_{2}$ ), the scattering reconstruction results are almost similar, and we cannot determine which of the two approximations is the most efficient approach. Here, the targets are not 'well resolved', strong multiple scattering exists, and the DWBA shows low performance. For higher levels of noise $\mathrm{n}_{2}$, there are spurious peaks that affect the scatterers detection (pseudospectra in Figs. 3a and 3c). By contrast, at a lower noise level (AWGN noise $\mathrm{n}_{1}$ ), FL is a better choice (Fig. 2). A comparison between the reconstructed and exact localised targets in a noisy environment indicates that not all scatterers are correctly reconstructed. More specifically, the scatterer locations are correctly indicated (as a group of scatterers) but the scattering amplitudes relative to the exact case (i.e., a single scatterer) show errors. The best target reconstruction and separation were provided for the diamond and ellipse geometries, as illustrated in Fig. 4. Under such circumstances, the SNR values dramatically decreased. The reported data suggest that the FoldyLax formulation is a better solution for solving inverse scattering problems and locating point targets. The pseudospectra provided by the FL approximation have larger signals. FL approximation feasibility was demonstrated by the average visibility index $\Upsilon$ distribution over the wavelength.

The results are in accordance with those reported by Ramm and Gutman [23]. They pointed out that there is no single approach to obtain a proper signal reconstruction using inverse scattering problems. Various solutions can be efficiently implemented for particular targets' spatial localisations. Baussard and Boutin [24] reported on an improved method called 'a recursive time-reversal MUSIC algorithm' devoted to improving the detection of closely placed targets. In noisy cases, the detection and the identification of the locations of the targets failed. Gruber et al. [17] pointed out that the MUSIC algorithm can locate targets beyond the DWBA approximation but under the assumption of a large number of targets relative to the number of sources. By contrast, our results indicate that MUSIC 
succeeds in detecting and localising targets placed in various spatial positions when the number of targets is lower than the number of transceivers. The proposed method corroborates all parameters and indicates that the diamond (D) and ellipse (E) geometries enable the best target localisation.

The numerical values gathered during the simulations showed that the efficiency of the target location depends slightly on the placement of the interior points, but it is strongly dependent on the wavelength and the level of noise. In paper [25] the problem of point scatterers reconstruction in a 2D space/waveguide using a MUSIC type algorithm connected with a factorization method for waveguides is reported. This method is applied for scatterers satisfying the Dirichlet and Neumann condition in the Born approximation. The reported numerical implementations are "sparse objects" for the case of $\mathrm{M}=3$, 4-point scatterers and $\mathrm{N}=10$. Small amounts of noise were used for both simulations and the reported reconstruction results were perfect.

Although the current simulation results are acceptable, there is a main source of uncertainty in the scattering measurements provided by uncertainties in the relationship between the scattering amplitude and the measurement of the scattering at a single angle, i.e. on the incident direction. The time computation is reasonable as it does not exceed $10 \mathrm{~s}$ for $3 \mathrm{D}$ representations despite the large number of parameters used in the simulation. However, some limitations were identified:

- when multiple scattering between the scatterers becomes important, the DWBA can no longer be employed;

- the requirement for a priori knowledge of the background environment and free-space/background Green's function as mentioned in [17];

- FL is restricted to the reciprocal media condition of the background and complete Green functions where $G_{0}\left(y_{r} y^{\prime}\right)-G_{0}\left(y_{r}^{2}, y\right)$ and $\left.G\left(y_{r} y^{\prime}\right)-G_{\left(y^{\prime}\right.}, y\right)$, but this condition does not severely restrict the generality of the MUSIC approach.

\section{CONCLUSIONS}

The numerical results showed a slight effectiveness of the Foldy-Lax approximation for scatterers location. We can preliminarily conclude that the inverse scattering problem is a proper approach for multiple target locations, particularly for targets in close proximity such as those having diamond and ellipse geometries with a target separation of $\lambda$ and $\sqrt{2}$. Improved results will be reported in the future.

Acknowledgments: The work of the first author was partially supported by the European Social Fund - the Sectoral Operational Programme Human Capital 2014-2020, through the Financial Agreement with the title „Burse pentru educaţia antreprenorială în rândul doctoranzilor şi cercetătorilor postdoctorat (Be Antreprenor!)" (in Romanian) or "Scholarships for entrepreneurial education among doctoral students and postdoctoral researchers (Be Entrepreneur!)", Contract no. 51680/09.07.2019 - SMIS code: 124539 .

\section{References}

1. Devaney, A.J.; Marengo, E.A.; Gruber, F.K. Time-reversal-based imaging and inverse scattering of multiply scattering point targets. J Acoust Soc Am 2005, 118, 3129-3138.

2. Marengo, E.A.; Hernandez, R.; Citron, Y.R.; Gruber, F.K.; Zambrano, M.; Lev-Ari, H. Compressive sensing for inverse scattering. In: XXIX URSI General Assembly, Chicago, Illinois, August 2008.

3. Miwa, T.; Arai, I. Super-resolution imaging for point reflectors near transmitting and receiving array. IEEE Trans Antennas Propagat 2004, 52: 220-229.

4. Necula (Stan), M.; Bibicu, D.; Moraru, L. Backscattering problems by a non-convex kiteshape objects in acoustic frequency domain. AIP Conference Proceedings 2071, 040008 (2019); https://doi.org/10.1063/1.5090075.

5. Kirsch, A.; Characterization of the shape of a scattering obstacle using the spectral data of the far field operator. Inverse Probl 1998, 14: 1489-1512. 
6. Devaney, A.J.; Dennison, M. Inverse scattering in inhomogeneous background media. Inverse Probl 2003; 19: 855.

7. Foldy, L.L.; The multiple scattering of waves. Pyhs Rev 1945, 67: 107-119.

8. Lax, M. Multiple scattering of waves. Rev. Mod. Phys. 1951, 23: 287-310.

9. Griesmaier, R.; Hanke M. MUSIC-characterization of small scatterers for normal measurement data. Inverse Probl 2009,25: 12.

10. Ammari, H.; Iakovleva, E.; Lesselier, D.; Perrusson, G. MUSIC-type electromagnetic imaging of a collection of small three-dimensional bounded inclusions. SISC 2007, 29: 674

11. Bibicu, D.; Necula (Stan), M..; Moraru, L. Acoustic radiation from baffled vibrating plates with various geometries - simulation results. AIP Conference Proceedings 2071, 040009 (2019); https://doi.org/10.1063/1.5090076.

12. Foldy, L. The multiple scattering of waves. I. General theory of isotropic scattering by randomly distributed scatterers. Phys Rev 1945, 67: 107-119.

13. Huang, K.; Li, P. A two-scale multiple scattering problem, Multiscale Model. J. Simul 2010,8: 1511-11534.

14. Huang, K.; Solna, K.; Zhao, H. Generalized Foldy-Lax formulation. J Comput Phys 2010, 229: 4544-4553.

15. Lev-Ari, H.; Devancy, A.J. The time-reversal technique re-interpreted: subspace-based signal processing for multi-static target location, Proceedings of the 2000 IEEE Sensor Array and Multichannel Signal Processing Workshop. SAM 2000, Cambridge, MA, USA

16. Kirsch, A. The MUSIC-algorithm and the factorization method in inverse scattering theory for inhomogeneous media. Inverse Probl 2002, 18(4): 1025.

17. Marengo, E.A.; Gruber, F.K. Subspace-Based Localization and Inverse Scattering of Multiply Scattering Point Targets. EURASIP J Adv in Sig Pr 2007, Article ID 17342 (16 pages).

18. Park, W-K. Interpretation of MUSIC for Location Detecting of Small Inhomogeneities Surrounded by Random Scatterers. Math Probl in Eng vol. 2016, Article ID 7872548 (13 pages)

19. Park, W-K. Asymptotic properties of MUSIC-type imaging in two-dimensional inverse scattering from thin electromagnetic inclusions SIAM J. Appl Math 2015, vol. 75, no.1, p. 209-228.

20. Park, W-K. Lesselier D. MUSIC-type imaging of a thin penetrable inclusion from its multistatic response matrix. Inverse Probl 2009, 25, (7), Article ID075002. (8 pages).

21. MathWorks. Available online: https://www.mathworks.com/help/signal/ref/spectrum.music.html (accessed on 11.03.2020).

22. Meilikov, E.; Farzetdinova, R. Color or Luminance Contrast - What is more Important for Vision? In: Advance in Neural Computation, Machine Learning and Cognitive Research, Eds. B. Kryzhanovski, W. Dunin-Barkowski, V. Redko, XIX Intl. Conf. on Neuroinformatics, 2017, Moscow, Russia, pp 147-156.

23. Ramm, AG.; Gutman, S. Optimization methods in direct and inverse scattering, Optimization Methods in Direct and Inverse Scattering. In: Continuous Optimization. Applied Optimization; Jeyakumar, V., Rubinov A., Eds.; Springer, Boston, 2005, vol. 99.

24. Baussard, A.; Boutin, T. Time-Reversal Music Imaging using a recursive approach. In: 15th European Signal Proceedings of Conference (EUSIPCO 2007), Poznan, Poland, September 3 7 2007, pp. 409-413.

25. Gintides, D.; Kiriaki, K.; Lygidaki, A.; Midrinos L. The detection of point scatterers in a waveguide, Advanced Topics in Scattering and Biomedical Engineering. In: Proceedings of the 8th Int. Workshop on Mathematical Methods in Scattering Theory and Biomedical Engineering, Lefkada, Greece, 2007, Eds A. Charalambopoulos, D.I Fotiadis and D. Polyzos, pp. 38-46. 